PROCEEDINGS OF THE

AMERICAN MATHEMATICAL SOCIETY

Volume 139, Number 6, June 2011, Pages 2163-2173

S 0002-9939(2011)10885-2

Article electronically published on February 4, 2011

\title{
INCOMING AND DISAPPEARING SOLUTIONS FOR MAXWELL'S EQUATIONS
}

\author{
FERRUCCIO COLOMBINI, VESSELIN PETKOV, AND JEFFREY RAUCH
}

(Communicated by Walter Craig)

\begin{abstract}
We prove that in contrast to the free wave equation in $\mathbb{R}^{3}$ there are no incoming solutions of Maxwell's equations in the form of spherical or modulated spherical waves. We construct solutions which are corrected by lower order incoming waves. With their aid, we construct dissipative boundary conditions and solutions to Maxwell's equations in the exterior of a sphere which decay exponentially as $t \rightarrow+\infty$. They are asymptotically disappearing. Disappearing solutions which are identically zero for $t \geq T>0$ are constructed which satisfy maximal dissipative boundary conditions which depend on time $t$. Both types are invisible in scattering theory.
\end{abstract}

\section{INTRODUCTION}

This paper is devoted to the construction of incoming solutions of Maxwell's equations in the exterior of a sphere which are totally absorbed at the boundary where a boundary condition defining a well-posed initial boundary value problem is imposed. By definition, disappearing solutions $u(t, x)$ vanish for $t \geq T_{0}>0$ and all $x$ outside a bounded obstacle. We also construct solutions whose total energy decays exponentially as $t \rightarrow+\infty$. We call such solutions asymptotically disappearing. For both types of solutions the scattered field vanishes identically. They are also of interest from the point of view of inverse problems as the information in the incoming wave is irretrievably lost (see 4 and Chapter IV in 5 , for more comments).

There are some classic examples. The first one is the equation

$$
\partial_{t} u-\partial_{x} u=0, \quad \text { in } x>0
$$

with no boundary condition imposed at $x=0$. Compactly supported data disappear in finite time, and all solutions with square integrable initial data tend to zero in $L^{2}(\{x>0\})$ as $t \rightarrow \infty$.

The wave equation in dimension $d=1$ with absorbing boundary condition is

$$
\partial_{t}^{2} u-\partial_{x}^{2} u=0 \quad \text { in } x>0, \quad \partial_{t} u(t, 0)-\partial_{x} u(t, 0)=0 .
$$

Received by the editors June 12, 2010.

2010 Mathematics Subject Classification. Primary 35Q61; Secondary 35P25, 35L45.

Key words and phrases. Maxwell equations, disappearing solutions, dissipative boundary conditions.

The third author's research was partially supported by the National Science Foundation under grant NSF DMS 0405899.

(C)2011 American Mathematical Society Reverts to public domain 28 years from publication 
The standard energy is decreasing in time, and leftward moving waves which satisfy $\partial_{t} u-\partial_{x} u=0$ are absorbed at the boundary without reflection.

In these two cases a significant fraction of solutions disappear. The situation is radically different for the wave equation in dimensions $d>1$ where it is not easy to produce a single example that disappears, that is, to find a solution $u(t, x)$ which vanishes for $t \geq T_{0}>0$. The classic example is in the fundamental paper of Majda 4. He considers for the spatial dimension $d=3$ the differential equation and boundary condition

$$
\partial_{t}^{2} u-\Delta u=0 \quad \text { in } r:=|x| \geq R, \quad\left(\partial_{t}-\partial_{r}\right)(r u)=0 \quad \text { on }|x|=R .
$$

All incoming spherical waves

$$
u(t, x)=\frac{f(|x|+t)}{|x|}
$$

satisfy the boundary condition $\left(\partial_{t}-\partial_{r}\right)(r u)=0$. Choosing $f \in C_{0}^{\infty}(\mathbb{R})$ yields a disappearing solution. We observe that the natural absorbing boundary condition $\partial_{t} u-\partial_{r} u=0$ is satisfied modulo a correction $-f(|x|+t) /|x|^{2}$. The modified boundary condition is satisfied exactly. A similar phenomenon will be described for Maxwell's system in Section 3.

For Maxwell's equations,

$$
\begin{gathered}
E_{t}=\operatorname{curl} B, \quad B_{t}=-\operatorname{curl} E, \\
\operatorname{div} E=\operatorname{div} B=0,
\end{gathered}
$$

Georgiev 2] constructed, using spherical coordinates, disappearing fields which are spherical waves modulated in the angular direction; that is, $E$ and $B$ are of the form

$$
\frac{f(|x|-t, x /|x|)}{|x|} .
$$

His solution is singular along the $z$-axis $\{x=y=0\}$, where it is locally integrable and so defines a well-defined distribution. The fields satisfy Maxwell's equations on the complement of the $z$-axis. We prove below that they are not solutions in a neighborhood of this axis. In Section 2 we prove that Maxwell's equations have no solutions with the structure of modulated spherical waves. In Section 3 we construct incoming solutions for Maxwell's equations which have a similar form, namely,

$$
\frac{f_{1}(t+|x|, x /|x|)}{|x|}+\frac{f_{2}(t+|x|, x /|x|)}{|x|^{2}}+\frac{f_{3}(t+|x|, x /|x|)}{|x|^{3}} .
$$

As for the wave equation, the natural absorbing condition

$$
E_{\tan }-n(x) \wedge B_{\tan }=0, \quad|x|=1,
$$

$n(x)$ being the unit normal to $|x|=1$ pointing into $|x| \leq 1$, is not satisfied. In Theorem 4.1 we construct asymptotically disappearing solutions satisfying a small perturbation of this maximal dissipative boundary condition. With small and time dependent perturbations we construct disappearing solutions in Theorem 4.2. The problem of constructing a disappearing solution of a time independent dissipative boundary value problem for Maxwell's equations remains open. 


\section{No modulated SPherical Maxwell SOlutions}

The large time asymptotics for solutions of the wave equation on $\mathbb{R}^{1+3}$ with Cauchy data in the Schwartz space $\mathcal{S}\left(\mathbb{R}^{3}\right)$ is given in terms of a profile $h(s, \omega) \in$ $\mathcal{S}\left(\mathbb{R} \times \mathbb{S}^{2}\right)$ as

$$
u=\frac{h(|x|-t, x /|x|)}{|x|}+\mathcal{O}\left(|x|^{-2}\right)
$$

The expression $h(|x|-t, \omega) /|x|$ is for each $\omega$ an outgoing spherical wave solution of the wave equation in $\{x \neq 0\}$.

Consider next Maxwell's equations (1.1), (1.2). They imply that

$$
\square E=\square B=0 .
$$

For Cauchy data in $\mathcal{S}$ the asymptotics are given in terms of profiles

$$
\mathbf{e}(s, \omega) \text { satisfying } \quad \omega \cdot \mathbf{e}=0, \quad \mathbf{b}(x, \omega):=\omega \wedge \mathbf{e} .
$$

As $t \rightarrow+\infty$,

$$
(E(t, x), B(t, x))=\left(\frac{\mathbf{e}(|x|-t, x /|x|)}{|x|}, \frac{\mathbf{b}(|x|-t, x /|x|)}{|x|}\right)+\mathcal{O}\left(\frac{1}{t^{2}+|x|^{2}}\right) .
$$

The second condition $\omega \cdot \mathbf{e}=0$ asserts that for each $s, \omega \mapsto \mathbf{e}(s, \omega)$ is a tangent vector field on $\mathbb{S}^{2}$. Thus as soon as it is continuous, Brouwer's Theorem implies that there is at least one $\underline{\omega} \in \mathbb{S}^{2}$ where $\mathbf{e}(s, \underline{\omega})=\mathbf{b}(s, \underline{\omega})=0$. There are always quiet spots in the far field.

In analogy with the spherical wave solutions of the wave equation it is natural to ask if there are some special profiles so that

$$
(E, B):=\left(\frac{\mathbf{e}(|x|-t, x /|x|)}{|x|}, \frac{\mathbf{b}(|x|-t, x /|x|)}{|x|}\right)
$$

exactly satisfies Maxwell's equations in $\{x \neq 0\}$. With

$$
w(t, r, \omega):=\left(\frac{\mathbf{e}(r-t, \omega)}{r}, \frac{\mathbf{b}(r-t, \omega)}{r}\right),
$$

this is an expression of the form

$$
w(t,|x|, x /|x|), \quad \text { where } \quad \square_{t, x} w(t,|x|, \omega)=0 .
$$

We next prove that it is impossible for fields as in (2.3) to satisfy Maxwell's equations unless they are independent of $x$. Thus,

- The leading expression in the asymptotic description is never an exact solution.

- There is no natural analogue for Maxwell's equations of exact spherical wave solutions of the wave equation.

- Georgiev's disappearing solutions [2] do not satisfy Maxwell's equations on the $z$-axis.

Suppose that $I$ is a nontrivial open interval in $\mathbb{R}, 0<a<b \leq \infty$, and $v$ is a distribution on $I \times] a, b\left[\times \mathbb{S}^{2}\right.$. If $v$ is smooth and $\psi \in C_{0}^{\infty}(I \times\{a<|x|<b\})$, the spherical coordinates $x=r \omega$ yield

$$
\langle v(t,|x|, x /|x|), \psi\rangle=\int_{I} \int_{a}^{b} \int_{\mathbb{S}^{2}} v(t, r, \omega) \psi(t, r \omega) r^{d-1} d t d r d \omega .
$$

Since the map

$$
C_{0}^{\infty}(I \times\{a<|x|<b\}) \ni \psi \quad \mapsto \quad \psi(t, r \omega) r^{d-1} \in C_{0}^{\infty}(I \times] a, b\left[\times \mathbb{S}^{2}\right)
$$


is continuous, it follows that $v(t,|x|, x /|x|)$ is a well-defined distribution on $I \times\{a<$ $|x|<b\}$ for any distribution $v \in \mathcal{D}^{\prime}(I \times] a, b\left[\times \mathbb{S}^{2}\right)$. Our first step concerns the wave equation.

Theorem 2.1. Suppose that $v \in \mathcal{D}^{\prime}\left(\{I \times] a, b\left[\times \mathbb{S}^{2}\right\}\right)$ is a radial solution of the wave equation as a function of $(t, x)$, that is,

$\square_{t, x} v(t,|x|, \omega)=0 \quad$ in the sense of distributions on $I \times\{a<|x|<b\} \times \mathbb{S}^{2}$.

Then the distribution $v(t,|x|, x /|x|)$ satisfies the wave equation on $I \times\{a<|x|<b\}$ if and only if $v$ is independent of $\omega$.

Proof. If $f(\omega)$ is a distribution on $\mathbb{S}^{2}$, then the distribution $f(x /|x|)$ on $\{x \neq 0\}$ has vanishing radial derivatives, so

$$
\Delta_{x}(f(x /|x|))=\left.|x|^{-2} \Delta_{\omega} f\right|_{\omega=x /|x|},
$$

where $\Delta_{\omega}$ denotes the Laplace-Beltrami operator on $\mathbb{S}^{2}$. Therefore

$$
\square_{t, x}(v(t,|x|, x /|x|))=\left.\square_{t, x} v(t,|x|, \omega)\right|_{\omega=x /|x|}-\left.|x|^{-2} \Delta_{\omega} v\right|_{\omega=x /|x|} .
$$

By hypothesis the first term vanishes and one concludes that $\left.\Delta_{\omega} v\right|_{\omega=x /|x|}=0$. Thus $v(t, r, \cdot)$ is harmonic as a function of $\omega \in S^{2}$, so is independent of $\omega$.

Theorem 2.2. Suppose that $w \in \mathcal{D}^{\prime}\left(I \times\left[a, b\left[\times \mathbb{S}^{2}: \mathbb{C}^{6}\right)\right.\right.$ satisfies

$$
\square_{t, x} w(t,|x|, \omega)=0 \quad \text { in } \quad I \times\{a<|x|<b\} \times \mathbb{S}^{2} .
$$

Then $u:=w(t,|x|, x /|x|):=(E(t, x), B(t, x))$ satisfies

$$
\square u=0 \quad \text { and } \quad \operatorname{div} E=\operatorname{div} B=0 \quad \text { in } I \times\{a<|x|<b\}
$$

if and only if there are constant vectors $c_{1}$ and $c_{2}$ so that $u=c_{1}+c_{2} t$.

Proof. Theorem 2.1 implies that $w$ is independent of $\omega$. Choose $\rho \in C_{0}^{\infty}(] 0,1[)$ with $\int \rho(s) d s=1$. For $1 \gg \varepsilon>0$ define

$$
w^{\varepsilon}:=\int w(t+\varepsilon s, r) \rho(s) d s \quad \text { and } \quad u^{\varepsilon}:=w^{\varepsilon}(t,|x|) .
$$

Since $u$ is the limit of the $u^{\varepsilon}$ in the sense of distributions, it suffices to prove that $w^{\varepsilon}$ is of the form $c_{1}^{\varepsilon}+c_{2}^{\varepsilon} t$.

Since $w^{\varepsilon}$ is smoothed in $t$, its wavefront set is contained in $\{(t, x, \tau, \xi): \tau=0\}$. Since it is a solution of the wave equation, the wavefront set is a subset of the characteristic variety $\left\{\tau^{2}=|\xi|^{2}\right\}$. Since these sets are disjoint in $\left\{(\tau, \xi) \in \mathbb{R}^{1+3} \backslash 0\right\}$, $w^{\varepsilon} \in C^{\infty}$. Thus it is sufficient to prove the result for smooth $w$ independent of $\omega$.

For such $w$ compute

$$
\operatorname{div} E(t,|x|)=\sum_{j=1}^{3} \frac{\partial E_{j}}{\partial r} \frac{\partial r}{\partial x_{j}} \quad \text { for } \quad a<|x|<b .
$$

Use $\operatorname{div} E=0$ and multiply by $2 r$ to find

$$
0=\sum_{j=1}^{3} \frac{\partial E_{j}}{\partial r} \frac{\partial r^{2}}{\partial x_{j}}=\sum_{j=1}^{3} \frac{\partial E_{j}(t, r)}{\partial r} 2 x_{j} .
$$

If $E$ is not independent of $r$, choosing $x$ parallel to $\partial_{r} E$ yields a contradiction. Therefore $E$ is independent of $r$. The same argument shows that $B$ is independent of $r$. 
Since $(E(t), B(t))$ satisfies the wave equation it must be a linear function of $t$.

The next corollary shows that there are no solutions of the dynamic equations alone, that is, without the divergence equations (1.2).

Corollary 2.3. If $w \in \mathcal{D}^{\prime}(I \times] a, b\left[\times \mathbb{S}^{2}\right)$ satisfies (2.5) and $u:=w(t,|x|, x /|x|)$ satisfies the dynamic Maxwell's equations (1.1), then there is a constant $c$ and a distribution $f \in \mathcal{D}^{\prime}(\{a<|x|<b\})$ so that $u=f(x)+c t$.

Proof. Denote $u=(E(t, x), B(t, x))$. The function $v=\partial_{t} u=(\operatorname{curl} B,-\operatorname{curl} E)$ is then of the form $\tilde{w}(t,|x|, x /|x|)$ and satisfies the dynamic equations and the divergence conditions (1.2). Theorem 2.2 implies that $v=v(t)$ is a linear function of $t$. Plugging this into the dynamic equation, one finds that $v$ must be constant.

Therefore $u_{t}$ is constant. It follows that $u=c t+f(x)$, with $f$ a distribution depending only on $x$.

\section{InCOMING SOLUTIONS OF MAXWELl's EQUATIONS}

Theorem 3.1. If $h \in C^{\infty}(\mathbb{R})$ and for all $k, \partial^{k} h(s) \in L^{1}([0, \infty[)$, then

$$
B:=-\left(\frac{h^{\prime \prime}}{|x|}-\frac{3 h^{\prime}}{|x|^{2}}+\frac{3 h}{|x|^{3}}\right) \frac{x}{|x|} \wedge\left(\frac{x}{|x|} \wedge(1,0,0)\right)+2\left(\frac{h^{\prime}}{|x|^{2}}-\frac{h}{|x|^{3}}\right)(1,0,0),
$$

where the argument of the functions $h^{(k)}$ is $|x|+t$, define smooth divergence free incoming solutions of Maxwell's equations in $\mathbb{R}_{t} \times\left(\mathbb{R}^{3} \backslash 0\right)$.

Proof. Since

$$
g(t, x):=\frac{f(|x|+t)}{|x|}
$$

is an incoming spherical solution of the wave equation,

$$
E=\operatorname{curl}(g, 0,0)=\left|\begin{array}{ccc}
i & j & k \\
\partial_{1} & \partial_{2} & \partial_{3} \\
g & 0 & 0
\end{array}\right|=\left(0, \partial_{3} g,-\partial_{2} g\right)
$$

is a divergence free incoming solution of the vector wave equation.

The derivatives $\partial_{j} g$ have two terms from the product rule,

$$
\partial_{j} g=\frac{f^{\prime}(|x|+t) \partial_{j}|x|}{|x|}-\frac{f(|x|+t) \partial_{j}|x|}{|x|^{2}}, \quad \partial_{j}|x|=\frac{x_{j}}{|x|} .
$$

Therefore,

$$
E=\left(\frac{f^{\prime}(|x|+t)}{|x|}-\frac{f(|x|+t)}{|x|^{2}}\right) \frac{x}{|x|} \wedge(1,0,0) .
$$

The vector field $(x /|x|) \wedge(1,0,0)$ on the sphere $|x|=1$ winds around the latitude lines which are the intersections with the planes $x_{1}=$ const.

Compute

$$
\partial_{t} E=\left(0, \partial_{t} \partial_{3} g,-\partial_{t} \partial_{2} g\right), \quad \partial_{t} \partial_{j} g=\frac{f^{\prime \prime}(|x|+t) \partial_{j}|x|}{|x|}-\frac{f^{\prime}(|x|+t) \partial_{j}|x|}{|x|^{2}}
$$


to find

$$
\partial_{t} E=\left(\frac{f^{\prime \prime}(|x|+t)}{|x|}-\frac{f^{\prime}(|x|+t)}{|x|^{2}}\right) \frac{x}{|x|} \wedge(1,0,0) .
$$

Since $x \wedge(1,0,0)=\left(0, x_{3},-x_{2}\right)$, one has

$$
x \wedge(x \wedge(1,0,0))=\left|\begin{array}{ccc}
i & j & k \\
x_{1} & x_{2} & x_{3} \\
0 & x_{3} & -x_{2}
\end{array}\right|=\left(-x_{2}^{2}-x_{3}^{2}, x_{1} x_{2}, x_{1} x_{3}\right) .
$$

Write

$\operatorname{curl} E=\operatorname{curl}\left(\varphi \frac{x}{|x|} \wedge(1,0,0)\right), \quad$ with $\quad \varphi:=\left(\frac{f^{\prime}(|x|+t)}{|x|}-\frac{f(|x|+t)}{|x|^{2}}\right)$.

Use the product rule

$$
\operatorname{curl}(\varphi \mathbf{w})=(\operatorname{grad} \varphi) \wedge \mathbf{w}+\varphi \operatorname{curl} \mathbf{w}
$$

to find

$$
\operatorname{curl} E=(\operatorname{grad} g) \wedge\left(\frac{x}{|x|} \wedge(1,0,0)\right)+g \operatorname{curl}\left(\frac{x}{|x|} \wedge(1,0,0)\right) .
$$

Next evaluate the curl term in (3.9). Identity (3.8) yields

$$
\operatorname{curl}\left(\frac{1}{|x|}\left(0, x_{3},-x_{2}\right)\right)=\operatorname{grad}\left(\frac{1}{|x|}\right) \wedge\left(0, x_{3},-x_{2}\right)+\frac{1}{|x|} \operatorname{curl}\left(0, x_{3},-x_{2}\right) \text {. }
$$

Since grad $|x|^{-1}=-|x|^{-2} x /|x|$, the first term in (3.10) is equal to

$\operatorname{grad}\left(\frac{1}{|x|}\right) \wedge(x \wedge(1,0,0))=-\frac{1}{|x|^{3}}(x \wedge(x \wedge(1,0,0)))=-\frac{1}{|x|} \frac{x}{|x|} \wedge\left(\frac{x}{|x|} \wedge(1,0,0)\right)$.

For the second term in (3.10), use

$$
\operatorname{curl}\left(0, x_{3},-x_{2}\right)=\left|\begin{array}{ccc}
i & j & k \\
\partial_{1} & \partial_{2} & \partial_{3} \\
0 & x_{3} & -x_{2}
\end{array}\right|=(-2,0,0) .
$$

Adding yields

$$
\varphi \operatorname{curl}\left(\frac{x}{|x|} \wedge(1,0,0)\right)=\left(-\frac{f^{\prime}}{|x|^{2}}+\frac{f}{|x|^{3}}\right) \frac{x}{|x|} \wedge\left(\frac{x}{|x|} \wedge(1,0,0)\right)-\frac{2 \varphi}{|x|}(1,0,0) .
$$

The field

$$
\frac{x}{|x|} \wedge\left(\frac{x}{|x|} \wedge(1,0,0)\right)=-(1,0,0)_{\tan }
$$

is equal to the tangential part of $(-1,0,0)$ on the sphere. It flows from the pole $x_{1}=-1$ to the opposite pole along the longitude lines.

The first summand in (3.9) is equal to

$$
\operatorname{grad} \varphi \wedge\left(\frac{x}{|x|}(1,0,0)\right)=\partial_{r} \varphi \frac{x}{|x|} \wedge\left(\frac{x}{|x|} \wedge(1,0,0)\right) .
$$


The derivative $\partial_{r} \varphi$ has terms where the derivative hits the $f$ factor and those when the derivative hits the $|x|^{-p}$. This yields

$$
\partial_{r} \varphi=\frac{f^{\prime \prime}}{|x|}-\frac{f^{\prime}}{|x|^{2}}-\frac{f^{\prime}}{|x|^{2}}+\frac{2 f}{|x|^{3}}=\frac{f^{\prime \prime}}{|x|}-\frac{2 f^{\prime}}{|x|^{2}}+\frac{2 f}{|x|^{3}} .
$$

So,

$$
\operatorname{grad} \varphi \wedge\left(\frac{x}{|x|}(1,0,0)\right)=\left(\frac{f^{\prime \prime}}{|x|}-\frac{2 f^{\prime}}{|x|^{2}}+\frac{2 f}{|x|^{3}}\right) \frac{x}{|x|} \wedge\left(\frac{x}{|x|} \wedge(1,0,0)\right) .
$$

Summing (3.14) and (3.18) yields

$$
\text { curl } E=\left(\frac{f^{\prime \prime}}{|x|}-\frac{3 f^{\prime}}{|x|^{2}}+\frac{3 f}{|x|^{3}}\right) \frac{x}{|x|} \wedge\left(\frac{x}{|x|} \wedge(1,0,0)\right)-2\left(\frac{f^{\prime}}{|x|^{2}}-\frac{f}{|x|^{3}}\right)(1,0,0) \text {. }
$$

Take $h \in \bigcap_{s} W^{s, 1}\left(\left[0, \infty[)\right.\right.$ as in the theorem and set $f=h^{\prime}$. Then the equation $B_{t}=-\operatorname{curl} E$ can be integrated using (3.19),

$$
\begin{aligned}
B & =-\int_{t}^{\infty}-\operatorname{curl} E(t, x) d t \\
& =\int_{t}^{\infty}\left(\frac{f^{\prime \prime}}{|x|}-\frac{3 f^{\prime}}{|x|^{2}}+\frac{3 f}{|x|^{3}}\right) \frac{x}{|x|} \wedge\left(\frac{x}{|x|} \wedge(1,0,0)\right)-2\left(\frac{f^{\prime}}{|x|^{2}}-\frac{f}{|x|^{3}}\right)(1,0,0) d t \\
& =\int_{t}^{\infty}\left(\frac{h^{\prime \prime \prime}}{|x|}-\frac{3 h^{\prime \prime}}{|x|^{2}}+\frac{3 h^{\prime}}{|x|^{3}}\right) \frac{x}{|x|} \wedge\left(\frac{x}{|x|} \wedge(1,0,0)\right)-2\left(\frac{h^{\prime \prime}}{|x|^{2}}-\frac{h^{\prime}}{|x|^{3}}\right)(1,0,0) d t \\
& =-\left(\frac{h^{\prime \prime}}{|x|}-\frac{3 h^{\prime}}{|x|^{2}}+\frac{3 h}{|x|^{3}}\right) \frac{x}{|x|} \wedge\left(\frac{x}{|x|} \wedge(1,0,0)\right)+2\left(\frac{h^{\prime}}{|x|^{2}}-\frac{h}{|x|^{3}}\right)(1,0,0) .
\end{aligned}
$$

By construction, $E$ and $B$ are divergence free, $\square E=0$, and $B_{t}=-\operatorname{curl} E$. We must prove that $E_{t}-\operatorname{curl} B=0$. Using the fact that $\operatorname{div} E=0$, compute

$$
\partial_{t}\left(E_{t}-\operatorname{curl} B\right)=E_{t t}-\operatorname{curl} B_{t}=E_{t t}+\operatorname{curl} \operatorname{curl} E=E_{t t}-\Delta E=0 .
$$

So, for each $x, E_{t}(t, x)-\operatorname{curl} B(t, x)$ is independent of $t$. The formulas for $E, B$ show that it tends to zero as $t \rightarrow \infty$. Therefore it vanishes identically. This completes the proof of the theorem.

\section{Disappearing SOlutions for MaXwell's equations}

We construct asymptotically disappearing solutions in $|x|>1$ that satisfy a homogeneous boundary condition

$$
u=(E, B) \in \mathcal{N}(x), \quad \text { on } \quad|x|=1 .
$$

Here $\mathcal{N}(x)$ is a four-dimensional linear subspace of $\mathbb{C}^{6}$ depending smoothly on $x$. Therefore, (4.1) represents two linear constraints on the boundary values.

Write the Maxwell equations in matrix form

$$
u_{t}+\sum_{j=1}^{3} A_{j} \partial_{j} u=0 .
$$

The $A_{j}$ are real symmetric matrices and for $0 \neq \xi \in \mathbb{R}^{3}, A(\xi):=\sum A_{j} \xi_{j}$ has rank equal to 4 . Denote by $n(x)$ the outward unit normal to the boundary. For solutions of Maxwell's equations in $|x|>0$ suitably smooth and small at infinity, one has

$$
\frac{d}{d t} \int_{|x|>1}|u|^{2} d x=\int_{|x|=1}\langle A(n(x)) u, u\rangle d \sigma .
$$


The theory of maximal dissipative boundary value problems of Friedrichs [1] as extended by Lax and Phillips [3] shows that a sufficient condition for $\mathcal{N}$ to define a well-posed mixed initial boundary value problem generating a contraction semigroup on $L^{2}(\{|x| \geq 1\})$ is that

$$
\operatorname{dim} \mathcal{N}=4 \text { and } \forall|x|=1, \quad \forall u \in \mathcal{N}(x), \quad\langle A(n(x)) u, u\rangle \leq 0 .
$$

Such spaces are called maximal dissipative. It follows that $\mathcal{N}(x) \supset \operatorname{Ker} A(n(x))$ for all boundary points $x$.

For any unit vector $n$ the eigenvalues of $A(n)$ are $-1,0,1$. The kernel is the set of $(E, B)$ with both $E$ and $B$ parallel to $n$. The condition that $\mathcal{N}$ contain the kernel is equivalent to saying that belonging to $\mathcal{N}$ is determined entirely by the tangential components $\left(E_{t a n}, B_{t a n}\right)$. The eigenspace corresponding to the eigenvalues \pm 1 is equal to

$$
E_{ \pm}:=\left\{(E, B): E_{t a n}= \pm n \wedge B_{t a n}\right\}
$$

The span of eigenspaces $E_{-} \oplus \operatorname{ker} A(n)$ with nonpositive eigenvalues satisfies the strict dissipativity identity

$$
\forall u \in E_{-} \oplus \operatorname{Ker} A(n), \quad\langle A(n) u, u\rangle=-\left\|u_{\text {tan }}\right\|^{2}=-\left\|E_{\text {tan }}, B_{\text {tan }}\right\|^{2} .
$$

The vector $\left(E_{t a n}, B_{t a n}\right)$ is the projection orthogonal to Ker $A_{n}$. The Poynting vector is $E \wedge B$ and satisfies $\langle A(n) u, u\rangle=(E \wedge B) \cdot n$.

Lemma 4.1. The solutions of Theorem 3.1 satisfy on each sphere

$$
E_{t a n}-n \wedge B_{t a n}=-\frac{h}{|x|^{3}}\left(\frac{x}{|x|} \wedge(1,0,0)\right) \text {. }
$$

Proof. On spheres centered at the origin, the solutions from Theorem 3.1 satisfy $E=E_{\text {tan }}$. For all fields, $n \wedge B_{\text {tan }}=n \wedge B$. By using (3.15), we find

$$
\begin{aligned}
n \wedge B_{\text {tan }} & =\left(\frac{h^{\prime \prime}}{|x|}-\frac{3 h^{\prime}}{|x|^{2}}+\frac{3 h}{|x|^{3}}\right)\left(\frac{x}{|x|} \wedge(1,0,0)\right)+2\left(\frac{h^{\prime}}{|x|^{2}}-\frac{h}{|x|^{3}}\right)\left(\frac{x}{|x|} \wedge(1,0,0)\right) \\
& =\left(\frac{h^{\prime \prime}}{|x|}-\frac{h^{\prime}}{|x|^{2}}+\frac{h}{|x|^{3}}\right)\left(\frac{x}{|x|} \wedge(1,0,0)\right) .
\end{aligned}
$$

Subtracting from $E$ completes the proof.

Lemma 4.2. There is an $\varepsilon_{0}>0$ so that for $|\varepsilon|<\varepsilon_{0}$ the boundary space

$$
\mathcal{N}_{\varepsilon}:=\left\{(E, B):(1+\varepsilon) E_{\text {tan }}=n \wedge B_{\text {tan }}\right\} \supset \operatorname{Ker} A_{n}
$$

is maximal dissipative. On $|x|=1$ it satisfies, with a constant $c>0$ independent of $\varepsilon_{0}, u$,

$$
\forall u \in \mathcal{N}_{\varepsilon}, \quad\langle A(n) u, u\rangle \leq-c\left\|u_{\text {tan }}\right\|^{2}=-c\left\|\left(E_{\text {tan }}, B_{\text {tan }}\right)\right\|^{2} .
$$

Proof. Write

$$
\begin{gathered}
\mathcal{N}_{\varepsilon} \ni u=\left(n \wedge B_{t a n}, B_{\text {tan }}\right)-\left(\varepsilon E_{t a n}, 0\right):=v+w \\
\langle A(n) u, u\rangle=\langle A(n) v, v\rangle+2\langle A(n) v, w\rangle+\langle A(n) w, w\rangle .
\end{gathered}
$$

Let $v=(\widetilde{E}, \widetilde{B})$. By definition, $v \in \mathcal{N}_{0}$ and the strict dissipativity of $\mathcal{N}_{0}$ implies that

$$
\begin{aligned}
\langle A(n) v, v\rangle & \leq-\left\|v_{\text {tan }}\right\|^{2}=-\left\|\widetilde{E}_{\text {tan }}\right\|^{2}-\left\|\widetilde{B}_{\text {tan }}\right\|^{2} \\
& =-\left\|n \wedge B_{\text {tan }}\right\|^{2}-\left\|B_{\text {tan }}\right\|^{2}=-\left\|(1+\varepsilon)^{-1} E_{\text {tan }}\right\|^{2}-\left\|B_{\text {tan }}\right\|^{2} .
\end{aligned}
$$


The next estimate is

$$
\begin{gathered}
|\langle A(n) w, w\rangle| \leq\left\|w_{\text {tan }}\right\|^{2}=\left\|\varepsilon E_{\text {tan }}\right\|^{2} \\
2|\langle A(n) v, w\rangle| \leq 2\left\|v_{\text {tan }}\right\|\left\|w_{\text {tan }}\right\| \leq \frac{1}{4}\left\|v_{\text {tan }}\right\|^{2}+4\left\|w_{\text {tan }}\right\|^{2} .
\end{gathered}
$$

Therefore

$$
\begin{aligned}
\left\langle A_{n} u, u\right\rangle & \leq-\frac{3}{4}\left\|v_{\tan }\right\|^{2}+5\left\|w_{t a n}\right\|^{2} \\
& =-\frac{3}{4}\left(\left\|(1+\varepsilon)^{-1} E_{t a n}\right\|^{2}+\left\|B_{t a n}\right\|^{2}\right)+5\left\|\varepsilon E_{t a n}\right\|^{2},
\end{aligned}
$$

and the proof is complete.

Combining (3.1) with Lemma 4.1 shows that the solutions of Theorem 3.1] satisfy

$$
(1+\varepsilon) E_{t a n}-n \wedge B_{t a n}=\left(\frac{\varepsilon h^{\prime \prime}}{|x|}-\frac{\varepsilon h^{\prime}}{|x|^{2}}-\frac{h}{|x|^{3}}\right)\left(\frac{x}{|x|} \wedge(1,0,0)\right) .
$$

Asymptotically disappearing solutions are constructed by choosing $h$ so that the right-hand side vanishes.

Theorem 4.1 (Asymptotically disappearing Maxwell solution). With $\varepsilon_{0}$ from the preceding lemma and $0<\varepsilon<\varepsilon_{0}$, define $2 r:=1-\sqrt{1+4 / \varepsilon}<0$ and $h(s):=$ $e^{r s}$. Then $(E, B)$ defined by (3.1) and (3.2) yield a divergence free solution of the maximal dissipative boundary value problem defined by the Maxwell equations in $|x|>1$ with maximal dissipative boundary condition

$$
(1+\varepsilon) E_{\text {tan }}-n \wedge B_{\text {tan }}=0, \quad \text { on } \quad|x|=1 .
$$

For each $\alpha$ there is a constant $C(\varepsilon, \alpha)$ so that $\left|\partial^{\alpha}(E, B)\right| \leq C h(t+|x|)$. In particular, the energy decays exponentially as $t \rightarrow \infty$.

Proof. The solutions of the ordinary differential equation $\varepsilon h^{\prime \prime}-\varepsilon h^{\prime}-h=0$ are $e^{\rho s}$ with $\rho$ satisfying $\varepsilon \rho^{2}-\varepsilon \rho-1=0$. The roots of the last equation are

$$
\rho=\frac{\varepsilon \pm \sqrt{\varepsilon^{2}+4 \varepsilon}}{2 \varepsilon}=\frac{1}{2}(1 \pm \sqrt{1+4 / \varepsilon}) .
$$

The minus sign yields $r$, and it follows that $h=e^{r s}$ is a solution. Equation (4.4) shows that the boundary condition is satisfied. The estimates are immediate consequences of the formulas.

Remark 4.1. The semigroup of contractions on $L^{2}$ defined by the maximal dissipative boundary value problem is of the form $e^{t G}$ with generator $G$ (see, for instance, Ch. III in [5]). The solutions in Theorem 4.1 have the form $e^{r t} \varphi(x)$ with $G \varphi=r \varphi$.

The preceding strategy yields profiles which decay exponentially and never have compact support. It yields only asymptotically disappearing solutions. The next computations show that it is possible to choose $h$ with compact support so that the resulting function satisfies a time dependent boundary condition. Let

$$
h(y):=\left\{\begin{array}{ll}
\exp \left(-\frac{1}{b^{2}-y^{2}}\right), & |y|<b, \\
0, & |y| \geq b>1,
\end{array} \quad h \in C_{0}^{\infty}(\mathbb{R}) .\right.
$$


Theorem 4.2 (Disappearing Maxwell solutions). For any $\delta \in] 0,1[$ and $h$ given by (4.5) we can choose $b>1$ and $\gamma(t) \in C_{0}^{\infty}(\mathbb{R})$ with $|\gamma|<\delta$ so that $(E, B)$ defined by (3.1) and (3.2) is a divergence free solution of Maxwell's equations in $t \geq 0,|x| \geq 1$ satisfying the maximal strictly dissipative boundary condition

$$
(1+\gamma(t)) E_{\tan }-n \wedge B_{\tan }=0 \quad \text { on } \quad|x|=1 .
$$

Proof. Compute with $b>1$ to be chosen below,

$$
\begin{gathered}
h^{\prime}(y)=\frac{-2 y}{\left(b^{2}-y^{2}\right)^{2}} \exp \left(-\frac{1}{b^{2}-y^{2}}\right), \quad|y|<b, \\
h^{\prime \prime}(y)=\frac{2\left(3 y^{4}-2\left(b^{2}-1\right) y^{2}-b^{4}\right)}{\left(b^{2}-y^{2}\right)^{4}} \exp \left(-\frac{1}{b^{2}-y^{2}}\right), \quad|y|<b .
\end{gathered}
$$

For the electric field on $|x|=1$ compute

$$
\begin{aligned}
h^{\prime \prime}(y)-h^{\prime}(y) & =\frac{2\left(3 y^{4}-2\left(b^{2}-1\right) y^{2}-b^{4}\right)+2 y\left(b^{2}-y^{2}\right)^{2}}{\left(b^{2}-y^{2}\right)^{4}} \exp \left(-\frac{1}{b^{2}-y^{2}}\right) \\
& :=\frac{Q(y, b)}{\left(b^{2}-y^{2}\right)^{4}} \exp \left(-\frac{1}{b^{2}-y^{2}}\right)=\frac{Q(y, b)}{\left(b^{2}-y^{2}\right)^{4}} h, \quad|y|<b .
\end{aligned}
$$

Since $Q(1,1)=4$ and $Q$ is continuous, there is a $\mu \in] 0,1]$ so that $Q(y, b) \geq 3$ on $[1,1+\mu] \times[1,1+\mu]$.

Now set $y=|x|+t,|x| \geq 1, t \geq 0$. For $1 \leq 1+t<b \leq 1+\mu$, equation (4.6) asserts that

$$
h(1+t)=\frac{\left(b^{2}-(1+t)^{2}\right)^{4}}{Q(1+t, b)}\left[h^{\prime \prime}(1+t)-h^{\prime}(1+t)\right]
$$

and on $|x|=1$ the right-hand side of (4.3) satisfies

$-h(1+t)\left(\frac{x}{|x|} \wedge(1,0,0)\right)=-\frac{\left(b^{2}-(1+t)^{2}\right)^{4}}{Q(1+t, b)} E_{\mathrm{tan}}:=-\gamma(t) E_{\mathrm{tan}}, 1 \leq 1+t \leq b$,

defining $\gamma \in C^{\infty}([0, b-1])$. Taking $b<1+\mu$ sufficiently close to 1 guarantees that $|\gamma(t)|<\delta / 2$ for $0 \leq t \leq b-1$.

For $|x|=1$ and $1 \leq 1+t<b$ the solution satisfies the maximal strictly dissipative boundary condition

$0=\left(E_{\tan }-n \wedge B_{\tan }\right)+h(1+t)\left(\frac{x}{|x|} \wedge(1,0,0)\right)=(1+\gamma(t)) E_{\tan }-\left(n \wedge B_{\tan }\right)$.

Extend $\gamma$ to an element of $C_{0}^{\infty}(\mathbb{R})$ with $|\gamma|<\delta$. Since the solution vanishes identically on $|x|=1$ for $t \geq b-1$, the boundary condition (4.7) is satisfied in $t \geq 0,|x|=1$.

\section{REFERENCES}

[1] K. O. Friedrichs, Symmetric positive linear differential equations, Comm. Pure Appl. Math. 11 (1958), 333-418. MR0100718 (20:7147)

[2] V. Georgiev, Disappearing solutions for dissipative hyperbolic systems of constant multiplicity, Hokkaido Math. J. 15 (1986), 357-385. MR866953 (88a:35145)

[3] P. D. Lax and R. S. Phillips, Local boundary conditions for dissipative symmetric linear differential operators, Comm. Pure Appl. Math. 13 (1960), 427-455. MR0118949 (22:9718) 
[4] A. Majda, Disappearing solutions for the dissipative wave equation, Indiana Univ. Math. J. 24 (1974/75), 1119-1133. MR0385330(52:6194)

[5] V. Petkov, Scattering Theory for Hyperbolic Operators, North-Holland Publishing Co., Amsterdam, 1989. MR 1028780(91e:35170)

Dipartimento di Matematica, Università di Pisa, Pisa, Italia

E-mail address: colombini@dm.unipi.it

Institut de Mathématiques de Bordeaux, 351, Cours de la Libération, 33405 Talence, FRANCE

E-mail address: petkov@math.u-bordeaux1.fr

Department of Mathematics, University of Michigan, Ann Arbor, Michigan 481091043

E-mail address: rauch@umich.edu 